\title{
A Psicologia no hospital geral: aspectos históricos, conceituais e práticos
}

\author{
Psychology within a general hospital: Historical, \\ conceptual, and practical aspects
}

\author{
Adriano Valério dos Santos AZEVÊDO' \\ Maria Aparecida CREPALDI ${ }^{1}$
}

\section{Resumo}

A atuação da Psicologia aplicada ao ambiente hospitalar é marcada por fatos históricos relevantes que contribuíram para a definição da área e delimitação da prática profissional. Este artigo objetivou apresentar os aspectos históricos, conceituais e práticos da Psicologia no hospital geral nos Estados Unidos da América e no Brasil. Esse tipo de atividade da Psicologia se iniciou na década de 1950 e, posteriormente, ocorreu o reconhecimento da especialidade Psicologia da Saúde. A prática ocorre por meio da avaliação e intervenção psicológica hospitalar com especificidades, dependendo da abordagem teórica utilizada. No que se refere aos aspectos conceituais, o acompanhamento psicológico hospitalar visa facilitar a adaptação e enfrentamento das situações vivenciadas pelo paciente hospitalizado, assim como prioriza a tríade: paciente, família e equipe de saúde.

Palavras-chave: Hospitalização; Psicologia da saúde; Psicologia hospitalar.

\begin{abstract}
The practice of applied Psychology in the hospital environment is marked by relevant historical facts that contributed to the definition and delimitation of this area of professional practice. This article aimed to present the practical, conceptual, and historical aspects of Psychology within a general hospital in the United States of America and in Brazil. The provision of psychology services within a general hospital began in the 1950s, and sometime later Health Psychology was recognizes as a specialty. Such practice involves hospital evaluation and specific psychological interventions, depending on the theoretical approach used. With regard to the conceptual aspects, professional psychological support aims to facilitate adaptation and help hospitalized patients cope with the challenges they face prioritizing the triad: patients, family, and healthcare team.
\end{abstract}

Keywords: Hospitalization; Health psychology; Hospital psychology.

\footnotetext{
1 Universidade Federal de Santa Catarina, Centro de Filosofia e Ciências Humanas, Programa de Pós-Graduação em Psicologia. Campus Universitário, Trindade, 88040-900, Florianópolis, SC, Brasil. Correspondência para/Correspondence to: A.V.S. AZEVÊDO E-mail: <adrianoazevedopsi@yahoo.com>.

Apoio: Coordenação de Aperfeiçoamento de Pessoal de Nível Superior.
} 
Diferentes terminologias são utilizadas para indicar a área de atuação do psicólogo em hospitais, as quais relacionam saúde e doença (American Psychological Association, 1980). A inserção do psicólogo no ambiente hospitalar representa uma estratégia da Psicologia da Saúde, que focaliza a atenção terciária e delimita um espaço físico para o campo de práticas com diversas possibilidades de atuação. Assim, a Psicologia no hospital geral se refere à atuação do psicólogo em uma instituição com pacientes que estão vivenciando a situação de adoecimento e hospitalização. A Política Nacional de Atenção Hospitalar considera o hospital um local adequado para as práticas de promoção da saúde, prevenção, tratamento de doenças e reabilitação, aspectos relacionados ao processo saúde-doença (Brasil, 2013).

Na literatura nacional, os autores destacam pontos importantes da Psicologia no hospital geral, sendo possível verificar a ênfase na definição da área (Angerami-Camon, 2002, 2010; Chiattone, 2006) e nos procedimentos de intervenção psicológica referentes à prática profissional (Amorim, 2006; Belar, 2000; Belar \& Deardoff, 1995; Campos, 1995; Knobel, Andreoli, \& Erlichman, 2008; Romano, 1990, 1999). No que se refere às atividades desenvolvidas pelo psicólogo nesse tipo de ambiente, estão citadas na produção científica: preparação psicológica de pacientes para cirurgias; assistência aos familiares de pacientes hospitalizados; e acompanhamento psicológico de pacientes com doenças crônicas como o câncer, por exemplo, e que serão submetidos aos procedimentos invasivos (Amorim, 2006; Angerami-Camon, 2010; Broering \& Crepaldi, 2011, 2013; Chiattone, 2006; Knobel et al., 2008). A delimitação dessas atividades facilita o desenvolvimento das práticas psicológicas.

Assim, considera-se relevante a contextualização para compreender de que maneira os psicólogos iniciaram suas atividades nos hospitais, quais instrumentos de avaliação psicológica e técnicas de intervenção são utilizados e quais fatos históricos contribuíram para a evolução da área. A sistematização dessas informações auxilia na formação de psicólogos e facilita o conhecimento de aspectos relativos à área. Dessa forma, verifica-se a neces- sidade de identificar a trajetória histórica, o objeto de estudo e as características dessa área de atuação.

Por conta disso, o presente artigo objetivou apresentar os aspectos históricos, conceituais e práticos da Psicologia no hospital geral nos Estados Unidos da América e no Brasil. Trata-se de um estudo teórico realizado por meio de consultas à literatura especializada. As seguintes informações foram selecionadas: surgimento da área, atribuições profissionais do psicólogo no hospital geral, dados históricos sobre a inserção da Psicologia no hospital geral, eventos científicos, subáreas e diretrizes para a avaliação e intervenção psicológica hospitalar. Foram analisadas as fontes documentais da American Psychological Association (1980) e do Conselho Federal de Psicologia (2000, 2001, 2007). O texto está organizado na seguinte sequência: 1) histórico da Psicologia no contexto hospitalar: apresentação de informações sobre a inserção da Psicologia no hospital geral, caracterizando as situações e os momentos históricos que contribuíram para o surgimento da área; e 2) aspectos conceituais e práticos: definição da área e especificidades da prática profissional relacionadas à avaliação e à intervenção psicológica hospitalar.

\section{Breve histórico da Psicologia no contexto hospitalar}

\section{Estados Unidos da América}

Nos Estados Unidos da América (EUA), a atuação do psicólogo no hospital geral ocorreu após o término da Segunda Guerra Mundial (1939-1945), quando foi identificada a necessidade da assistência psicológica para os militares, que apresentavam uma série de reações psíquicas no período de hospitalização, como distúrbios da sensopercepção, alterações no humor e agitação psicomotora (Pate \& Kohut, 2003). As atividades da Psicologia nos ambientes de saúde iniciaram-se com o propósito de identificar as repercussões psicológicas decorrentes do processo de adoecimento e consequente hospitalização, buscando estratégias para minimizar as alterações psíquicas e compreender a experiência da pessoa doente. 
Os procedimentos invasivos relacionados aos exames periódicos e às cirurgias, bem como os efeitos das medicações ocasionavam o surgimento de episódios depressivos nos pacientes hospitalizados. Dessa maneira, esse período foi muito importante para o acompanhamento psicológico hospitalar (Nogueira-Martins \& Frenk, 1980). Verificou-se que a situação de adoecimento e hospitalização representava um fenômeno complexo, o qual necessitava do entendimento de vários profissionais. Isso possibilitou a integração do psicólogo nas equipes multiprofissionais de saúde. Em 1970, o programa federal dos EUA - The Civilian Health and Medical Program of the Uniformed Services -, reconheceu as atividades desse profissional na área da saúde, mas somente em 1977 a aprovação da ata oficializou as práticas para todo o país (Dorken, 1993; Enright, Resnick, DeLeon, Sciara, \& Tanney, 1990).

Na década de 1970, ocorreu, nos EUA, uma série de questionamentos relacionados à atuação do psicólogo nos projetos que incluíam promoção da saúde, prevenção e tratamento de doenças. Em 1973, iniciaram-se as investigações para analisar as atribuições desse profissional no contexto da saúde e os dados destacaram sua importância, mas foi somente em 1978 que a American Psychological Association oficializou a Divisão 38. Esse fato marcou o surgimento da área denominada de Psicologia da Saúde (Straub, 2008).

O primeiro presidente da American Psychological Association, Joseph Matarazzo, destacou que a Psicologia da Saúde tem o objetivo de estudar a etiologia das doenças orgânicas, desenvolver intervenções direcionadas para a promoção da saúde, prevenção e tratamento de doenças, buscando o diálogo com os representantes governamentais para aprimorar as políticas públicas (Matarazzo, 1980). De acordo com Belar (1997), os Psicólogos da Saúde investigam as manifestações físicas e psicológicas vivenciadas pelos indivíduos em uma situação de doença. Esse profissional também atua na prevenção de doenças e manutenção das condições saudáveis por meio de intervenções educativas.

Nos EUA, a atuação do psicólogo nos diversos contextos (postos de saúde, hospitais e comunidades), com o foco no processo saúde e doença, foi reconhecida internacionalmente por Psicologia da Saúde. Em 1978, a Califórnia foi o primeiro estado a inserir psicólogos nas equipes de saúde, o que possibilitou o crescimento significativo da profissão nas instituições hospitalares (Dorken, 1993). Na década de 1980, a American Psychological Association regulamentou as práticas dos Psicólogos da Saúde por meio de documentos que tinham o objetivo de apresentar as principais diretrizes para a atuação da categoria (Enright, Resnick, Ludwigsen, \& DeLeon, 1993).

\section{A American Psychological Association} publicou, em 1985, um documento intitulado " $A$ Hospital Practice Primer for Psychologists", que apresentava informações sobre a estrutura dinâmica das instituições hospitalares, destacando as habilidades necessárias dos profissionais de saúde com o propósito de facilitar a inserção do psicólogo no contexto hospitalar. Destaca-se que, em 1991, outro documento foi publicado pela mesma associação, o "Guidelines on Hospital Privileges: Credentialing and Bylaws", o qual apresentava as orientações para o treinamento de psicólogos para a atuação nos hospitais com ênfase na delimitação das atividades relacionadas à avaliação e à intervenção psicológica (Enright et al., 1993). Foram discutidas as propostas para a implantação dos serviços de Psicologia nos hospitais gerais com o objetivo de promover a integração entre teoria, prática e pesquisa para avaliar os modelos de atuação em Psicologia da Saúde. Enright et al. (1993) destacam que os documentos da American Psychological Association contribuíram para a legitimação das práticas psicológicas no ambiente hospitalar, assim como para a publicação de pesquisas, com o surgimento do periódico Journal Health Psychology, em 1982, que, com reconhecimento internacional, publica pesquisas até os dias de hoje.

Brasil

No Brasil, a Psicologia da Saúde está fundamentada no princípio da integralidade, uma concepção dinâmica que enfatiza a inter-relação de aspectos envolvidos no processo saúde e doença (Mattos, 2003) e na interdisciplinaridade. Esses aspectos estabelecem diálogo e fundamentam estratégias alternativas nas práticas de atenção à 
saúde (Bonaldi, Gomes, Louzada, \& Pinheiro, 2007). A atuação do psicólogo no hospital geral, que representa uma especificidade da Psicologia da Saúde no setor terciário, iniciou-se na década de 1950 com poucos profissionais psicólogos. Havia, no país, profissionais com formação nas áreas das Ciências Humanas os quais eram responsáveis pela assistência psicológica aos pacientes hospitalizados. Entretanto, verificou-se a necessidade do surgimento dos cursos de graduação em Psicologia para delimitar a atuação do psicólogo nas instituições de saúde (Angerami-Camon, 2002).

As primeiras atividades foram realizadas por Matilde Néder em 1954 na clínica ortopédica e traumatológica do Hospital das Clínicas da Faculdade de Medicina da Universidade Federal de São Paulo (HC-FMUSP), considerada a pioneira na área (Angerami-Camon, 2002). O acompanhamento psicológico para as crianças, realizado por essa psicóloga pioneira, ocorria durante o período pré e pós-operatório de cirurgias na região cervical.

A partir de 1956, na unidade pediátrica do HC-FMUSP, Aydil Pérez-Ramos foi a psicóloga responsável pela assistência às crianças hospitalizadas, que apresentavam diferentes patologias, e aos seus familiares, os quais permaneciam na condição de acompanhantes (Angerami-Camon, 2002). Pérez-Ramos, vinculada à equipe multiprofissional, desenvolvia atividades de psicodiagnóstico e intervenção psicológica hospitalar, procedimentos que estavam sendo utilizados em uma fase inicial e foram aprimorados a partir de sua experiência prática, considerando a necessidade de elaboração dos princípios técnicos. Na literatura consultada, foi possível identificar os estudos da autora acerca da criança hospitalizada (Queiroz, 1958, 1961).

Na década de 1950 verificou-se que a hospitalização infantil representou uma temática relevante para os psicólogos em hospitais e, desse modo, a atuação profissional contribuiu para o desenvolvimento de estudos científicos. Em 1974, o HC-FMUSP possibilitou a inserção da Psicologia no hospital geral nos Institutos de Ortopedia, Psiquiatria, Neurologia e da Criança. Além disso, impulsionou os serviços da Psicologia nos hospitais de especialidades (Instituto do Coração, Instituto Cen- tral, Instituto de Reabilitação), autorizou a contratação de profissionais psicólogos sob a direção de psicólogos, gerou manuais de trabalho, promoveu a participação de psicólogos nas equipes multiprofissionais por meio de tarefas delimitadas e compartilhadas e descreveu o perfil profissional para atuação na área. Um ponto a ser considerado foi a atuação de psicólogos nesses serviços nos hospitais, o que antes era restrito aos profissionais médicos do Hospital das Clínicas (HC).

Na década de 1970, a atuação do psicólogo no hospital geral representou uma temática de estudo na universidade. A Profa. Dra. Thereza Pontual de Lemos Mettel, da Faculdade de Medicina de Ribeirão Preto da Universidade de São Paulo (FMRP-USP), investiu na organização de uma equipe para a realização do trabalho clínico junto às crianças, o qual se consolidou e resultou em grande sucesso em tratamentos médicos de diversas especialidades. Depois, como professora da Universidade de Brasília, teve um papel importante na introdução de uma política de atenção hospitalar destinada à criança, que incluía os familiares acompanhantes e a participação ativa das mães no cuidado à saúde do filho. Realizou uma pesquisa no Hospital de Base do Distrito Federal, que mostrou melhora nas condições de saúde das crianças e redução do tempo de internação quando as mães permaneciam no local. Esses resultados culminaram na adoção de políticas de humanização em hospitais da rede pública de saúde (Mettel, 2007).

O trabalho da referida professora em $\mathrm{Ri}$ beirão Preto deu origem a um programa pioneiro destinado ao treinamento de psicólogos no hospital geral, considerado referência em Psicologia da Saúde no Brasil. Recebeu a denominação de Programa de Aprimoramento, apresentando a estrutura de um curso de residência, no qual o recém-formado desenvolvia atividades em tempo integral. Os psicólogos e professores Edna Maria Marturano e Ricardo Gorayeb foram responsáveis pela direção desse serviço, que incluía atividades de ensino, prática e pesquisa na área da Psicologia Clínica e Hospitalar no Hospital das Clínicas da FMRP-USP (Gorayeb, 2010). A partir de então, foram criados, no país, outros Programas de Aprimoramento Pro- 
fissional e, em 1976, o Ministério da Saúde iniciou a elaboração de Programas de Residência para Áreas da Saúde, nos quais constava a Psicologia. Entretanto, a proposta não apresentou avanços e foi arquivada. Posteriormente, ocorreu o surgimento das Residências Multiprofissionais e em Área Profissional da Saúde, por meio da Lei no 11.129 de 2005, na qual foi incluída a Psicologia pelo fato de ser considerada profissão da área da saúde (Brasil, 2005). Em 2007, foi publicada a Portaria Interministerial $n^{\circ} 45$ referente a essas residências e, em seguida, foi institucionalizada uma Comissão Nacional de Residência Multiprofissional em Saúde - Portaria Interministerial $n^{\circ}$ 698, de 19 de julho de 2007 (Brasil, 2007). A partir disso, o Conselho Federal de Psicologia (2007) apresentou as normas para o credenciamento de Programas de Residência em Psicologia na área da saúde (Resolução Conselho Federal de Psicologia n 15/2007).

É preciso considerar, ainda, o trabalho de Célia Zannon, que iniciou sua trajetória como psicóloga no Instituto da Criança do Hospital das Clínicas de São Paulo (HC-SP) e elaborou uma tese de doutorado pioneira na área de Psicologia hospitalar: "O comportamento de crianças hospitalizadas e a rotina hospitalar: subsídios para atuação do psicólogo junto à equipe de pediatria" (Zannon, 1981), sob a orientação da Profa. Dra. Thereza Mettel. Essa tese marcou o advento da pesquisa empírica em Psicologia em hospital geral.

Avanços significativos marcaram o crescimento da Psicologia no hospital geral devido à iniciativa de profissionais da área, os quais buscaram delimitar as práticas psicológicas. Na tentativa de facilitar a formação profissional, surge, em 1977, o primeiro curso de Psicologia Hospitalar realizado no país, na Pontifícia Universidade Católica de São Paulo, produzido e ministrado por Bellkiss Romano. A partir da década de 1980, destacam-se nessa trajetória o "I e o II Encontros Nacionais de Psicólogos da Área Hospitalar", eventos científicos que contribuíram para o aprimoramento profissional (Romano, 1999). A possibilidade de atuação do psicólogo nos ambientes de saúde representou, então, um período marcado por inúmeros questionamentos acerca das tarefas desse profissional, as quais precisavam ser definidas com clareza para orientar as práticas. De acordo com Santos e Jacó- Vilela (2009), os psicólogos estavam diante de teorias e técnicas das abordagens psicológicas, mas na tentativa de responder com urgência às demandas da instituição de saúde apresentavam dificuldades em estabelecer parâmetros para a atuação qualificada. Nas primeiras atividades da Psicologia implementadas em hospital geral, predominava uma perspectiva prática da Psicologia clínica tradicional. Diante das características da instituição hospitalar, a simples transposição do modelo mostrava-se insuficiente para atender uma demanda tão complexa. Assim, foi necessário promover uma reflexão crítica acerca das atribuições profissionais do psicólogo que atua em hospitais, principalmente no que se refere à sua inserção nas equipes de saúde, o que ocorreu de forma gradativa a partir do enfrentamento das dificuldades de sua inserção em um campo exclusivo das ciências biomédicas.

Após um período de enfrentamento desses desafios, nas décadas de 1970, 1980 e 1990, foram implantados os Serviços de Psicologia Hospitalar, que se tornaram referência no Brasil. Em 1974, o Serviço de Psicologia do HC-FMRP-USP já estava consolidado (Gorayeb, 2010), quando, no mesmo ano, foi implantado o Serviço de Psicologia do Instituto do Coração do HC-FMUSP (Romano, 1999). Em 1981, foi implantado o Serviço de Psicologia na Unidade de Pediatria do Hospital Universitário da Universidade Federal de Santa Catarina (Crepaldi, Gonçalves, \& Moré, 2011). No mesmo ano, o Serviço de Psicologia do Hospital de Base de São José do Rio Preto, no interior de São Paulo, foi implantado também por meio da iniciativa de psicólogos da Unidade de Pediatria (Miyazaki, Domingos, \& Valério, 2006). Em 1982, o Serviço de Psicologia do Hospital Brigadeiro, São Paulo, foi implantado por Heloísa Chiattone, priorizando o atendimento psicológico às crianças hospitalizadas e aos acompanhantes (Dias, M. N. Baptista, \& Baptista, 2003). Em 1986, foi institucionalizado o Serviço de Psicologia do Hospital de Clínicas de Porto Alegre, reconhecendo o trabalho das psicólogas que lá atuavam desde a década de 1970 (Silva, 2006). Já 
a Santa Casa de Misericórdia de São Paulo implantou o Serviço de Psicologia em 1992 (Bruscato, 2004).

O surgimento desses Serviços atendeu às demandas de crianças e adolescentes e contribuiu para legitimar as práticas psicológicas no território brasileiro. Outros tantos serviços foram implementados pelo país atendendo às necessidades de saúde de usuários nos diferentes momentos do ciclo de vida. Diante do crescimento dessa área, tornou-se necessário estabelecer diretrizes para a atuação profissional, considerando que os psicólogos precisavam compartilhar experiências e apresentar resultados de pesquisas.

No que se refere à produção da literatura especializada na área da Psicologia Hospitalar, Angerami-Camon $(1984,2002)$ iniciou a publicação de livros com o propósito de apresentar as possibilidades de atuação. Ocorreu o surgimento gradual dos cursos de especialização e a inserção da disciplina de Psicologia Hospitalar nos cursos de graduação (Sebastiani, 2006), iniciativas que foram relevantes para a formação profissional. Os psicólogos interessados na área da saúde procuravam aprimorar habilidades nesses cursos e nos Programas de Aprimoramento.

Em 1987, uma pesquisa sobre a atuação do psicólogo no hospital geral apontou que a Psicologia Hospitalar estava em uma fase de desenvolvimento inicial e os psicólogos precisavam estruturar o modelo de intervenção e as habilidades de ensino e pesquisa (Romano, 1999). Em síntese, no final da década de 1980, as estratégias de atuação ainda necessitavam de esclarecimentos acerca dos procedimentos técnicos, principalmente diante das diversas abordagens teóricas utilizadas pelos psicólogos nos hospitais. Romano (1990) organizou uma coletânea de textos sobre a prática da Psicologia nessas instituições, tratando de temáticas como: a importância do psicólogo identificar as repercussões psíquicas do paciente submetido à cirurgia coronariana; considerações acerca da atuação desse profissional nos centros de terapia intensiva; a intervenção psicológica com os pacientes fora de possibi-

578 lidades terapêuticas (em processo terminal); e espe- cificidades de pacientes com lesão medular, fissura de lábio e hipertensão arterial. Os textos apresentavam indicações para a atuação do psicólogo hospitalar e a produção bibliográfica repercutiu diretamente no surgimento de subáreas. Desse modo, as práticas psicológicas relacionadas à cardiologia e psico-oncologia se destacaram devido à inserção crescente de psicólogos nessas equipes de saúde.

Alguns acontecimentos históricos contribuíram para o desenvolvimento da Psicologia Hospitalar. Em 1994, a Sociedade Brasileira de Psico-Oncologia foi inaugurada para discutir as diretrizes teóricas da especialidade (Gimenes, Magui, \& Carvalho, 2006), pois representava uma área de interesse para os psicólogos que atuavam nos hospitais gerais. Em 1997, foi estabelecida a Sociedade Brasileira de Psicologia Hospitalar, a qual iniciou a publicação de um periódico em 2004, promovendo a integração de psicólogos nas reuniões científicas (Romano, 1999).

As atividades desses profissionais nos hospitais foram reconhecidas pelo Ministério da Saúde por meio de documentos que regulamentam o atendimento em Psicologia nos procedimentos de média e alta complexidade. Alguns exemplos são a obrigatoriedade dos serviços de Psicologia nas Unidades de Terapia Intensiva, na assistência à gestante de alto risco, nos centros de atendimento em oncologia, nas unidades de internação em hospital geriátrico, atendimento hospitalar de pacientes crônicos, pacientes em tratamento da obesidade e nas unidades de assistência em alta complexidade cardiovascular (Conselho Regional de Psicologia, 2007). Verificou-se o comprometimento das políticas públicas de saúde e os psicólogos demonstraram que ações efetivas são desenvolvidas nas instituições hospitalares. Destaca-se que o psicólogo hospitalar continuou buscando ampliar o campo de atuação. Um aspecto central se refere à prática profissional, a qual passou por um processo de aprimoramento e, embora muitas portarias apontem para a necessidade dos serviços de Psicologia em hospitais, muito ainda está por ser feito.

O atendimento psicológico hospitalar focaliza as repercussões psíquicas do indivíduo referentes 
à situação de doença e hospitalização. Busca-se investigar a capacidade de adaptação do paciente, os problemas vivenciados nesse ambiente, o nível de adesão ao tratamento e o relacionamento estabelecido entre paciente, acompanhante e equipe de saúde (Romano, 1999). A adaptação está ligada a uma concepção evolucionista do ser humano, ou seja, refere-se à capacidade do indivíduo de construir estratégias para o enfrentamento das situações que, a curto ou longo prazo, possibilitem um funcionamento produtivo, permitindo desenvolver recursos úteis para o seu crescimento pessoal (Leahy, Tirch, \& Napolitano, 2013). Inicia-se problematizando a situação para que as reflexões possam facilitar o surgimento de ideias as quais o auxiliem a enfrentar os acontecimentos, o que representa um aspecto importante para o indivíduo hospitalizado.

Essas colocações sobre a realidade hospitalar contribuíram para a delimitação das atividades profissionais e possibilitaram o surgimento dos grupos de estudo com o objetivo de aprimorar conhecimentos para auxiliar a prática do psicólogo hospitalar. No início do ano 2000, um grupo de psicólogos, sob a coordenação de Célia Zanonn, institucionalizou o Grupo de Trabalho em Psicologia Pediátrica da Associação Nacional de Pesquisa e Pós-Graduação em Psicologia (ANPEPP), promovendo a realização de pesquisas referentes à temática (Crepaldi, Rabuske, \& Gabarra, 2006).

Destaca-se que desde a década de 1950 os estudos sobre a criança hospitalizada despertavam interesse nos psicólogos que estavam iniciando as atividades nos hospitais. Esse grupo é responsável pela maioria dos trabalhos científicos publicados na área de Psicologia Pediátrica. A ANPEPP agregou outros grupos, como o Grupo de Trabalho em Psicologia da Saúde, coordenado pela professora Raquel Kerbauy (Universidade de São Paulo), contribuindo para a formação de psicólogos para a pesquisa na área da saúde, principalmente com a divulgação de estudos realizados no Laboratório de Saúde e Comportamento do Departamento de Psicologia Experimental da USP (Kerbauy, 1999). Atualmente, esse grupo recebe o nome de Grupo de Psicologia da Saúde em Instituições e na Comunidade e é coordenado pelos professores Ricardo Gorayeb (USP-Ribeirão Preto), Eliane Fleury Seidl (Universidade de Brasília) e Maria Cristina Miyazaki (Faculdade de Medicina de São José do Rio Preto - FAMERP). A professora Vera Lúcia Raposo do Amaral, da Pontifícia Universidade Católica de Campinas, contribuiu para o desenvolvimento de intervenções na área da saúde baseadas em evidências por meio das atividades de ensino e pesquisa, as quais promoveram avanços na maneira de sistematizar as práticas.

Os estudos teóricos e relatos de pesquisas empíricas com temáticas da Psicologia Hospitalar representaram um avanço importante para a divulgação do conhecimento científico, o qual tem sido considerado referência na área. Diversos estudos publicados por volta da década de 2000 contribuíram para o desenvolvimento de intervenções psicológicas relacionadas à pediatria (Crepaldi, 1999a,1999b; Dias et al., 2003; Crepaldi et al., 2006), cardiologia (Romano, 2001), câncer infantil (Valle, 2001) e obstetrícia (Bortoletti et al., 2007). Verificou-se a publicação de um livro sobre temas de ensino e pesquisa com a apresentação de exemplos de intervenções (Ismael, 2005); o manual de Psicologia Hospitalar com uma abordagem psicanalítica (Simonetti, 2004); e o manual de Psicologia Clínica para hospitais (Romano, 2008). Estes últimos discutem as principais tarefas do psicólogo em hospitais.

Além disso, Miyazaki et al. (2006) organizaram a publicação de pesquisas que auxiliaram a atuação profissional nos seguintes pontos: avaliação psicológica, preparação de crianças para cirurgias, identificação de estresse e estratégias de enfrentamento em pacientes submetidos a transplantes e hospitalizados por doenças crônicas e outras temáticas relevantes. Os estudos científicos contribuíram para o crescimento da área e reconhecimento das atividades profissionais do psicólogo no hospital geral. Isso possibilitou o desenvolvimento de Programas de Pós-Graduação stricto sensu (mestrado e doutorado); o Programa de Psicologia e Saúde da FAMERP; e o Programa Psicologia da Saúde da Universidade Estadual da Paraíba e da Universidade Metodista de São Paulo. Esses programas cumprem o objetivo de formar pesquisadores e docentes na área. 


\section{Psicologia no hospital geral: aspectos conceituais e práticos}

Na produção científica verificou-se que a definição da Psicologia no hospital geral é apresentada nos manuais específicos de autores brasileiros (Angerami-Camon, 2010; Chiattone, 2006; Romano, 1990, 1999) e a literatura internacional destaca as atividades práticas relacionadas à Psicologia da Saúde (Belar, 2000). A terminologia Psicologia Hospitalar, utilizada apenas no Brasil, destaca a atuação do psicólogo no hospital geral, embora represente um termo inadequado para se referir ao local de práticas de uma área profissional. Psicologia da Saúde é o termo correto para destacar as atividades desenvolvidas pelo psicólogo nos diversos contextos da área, neste caso, a atuação da Psicologia no hospital representa uma sub-especialidade da Psicologia da Saúde.

A especialidade Psicologia Hospitalar foi reconhecida pelo Conselho Federal de Psicologia (2000), por meio da Resolução nº 014/2000, na qual apresenta instruções para o psicólogo obter o registro. Os profissionais que atuavam nessa área solicitavam o registro de especialista após a conclusão dos cursos de especialização credenciados pelo Conselho Federal de Psicologia ou diante da comprovação de experiência prática de dois anos e aprovação nas provas teóricas. O Conselho Federal de Psicologia (2001), por meio da Resolução n 02/2001, definiu os parâmetros para a atuação na área, considerando relevante a avaliação e o acompanhamento psicológico aos pacientes hospitalizados e seus familiares com a utilização das teorias e técnicas adequadas. A resolução destaca que o psicólogo hospitalar desenvolve diferentes tipos de intervenção, atende pacientes que se encontram em ambientes distintos (como a unidade de terapia intensiva, enfermarias, ambulatórios etc.) e aponta que os procedimentos utilizados precisam priorizar a relação paciente, família e equipe de saúde por meio do contato interdisciplinar com os profissionais para compartilhar informações úteis para o direcionamento de estratégias.

A perspectiva interdisciplinar por meio do diálogo constante entre a equipe de saúde repre- nicação, conforme foi destacado por Chiattone (2006) e Almeida (2000). Isso possibilita discutir características de um caso clínico com os profissionais, definir procedimentos de intervenção e acompanhar os resultados avaliando seus efeitos.

Simonetti (2004) destaca, em uma perspectiva psicanalítica, que a Psicologia hospitalar focaliza, em um primeiro nível, a identificação dos pensamentos e sentimentos do indivíduo hospitalizado para, em seguida, iniciar o tratamento por meio de técnicas psicológicas. Segundo o autor, é preciso compreender as alterações emocionais vivenciadas pelos pacientes hospitalizados diante de uma situação de luto proveniente do surgimento da doença, assim será possível acompanhar o indivíduo no processo de elaboração dessa experiência por meio da exploração das verbalizações de maneira que o manejo da resistência e da transferência sejam fundamentais. Angerami-Camon (2010), a partir de uma fundamentação fenomenológica existencial, enfatiza que se trata de uma área a qual visa minimizar, no paciente, o sofrimento gerado pelo processo de hospitalização, por meio da escuta e da empatia para iniciar a ressignificação ou atribuiç̧ão de novos significados às suas vivências.

É importante ressaltar que outras perspectivas teóricas contextualizaram a sua capacidade de produzir conhecimentos no contexto hospitalar. Nesse sentido, podem ser citados autores da teoria sistêmica, os quais reconhecem a possibilidade do desenvolvimento de intervenções psicológicas pelo fato de considerarem o hospital um sistema aberto e dinâmico, repleto de significados nas relações que são estabelecidas entre paciente, família e equipe de saúde. Essa visão multidimensional, focalizada na comunicação, na complexidade do contexto hospitalar e na intersubjetividade, representa o elemento central para reflexões críticas (Moré, Crepaldi, Gonçalves, \& Menezes, 2009). Psicólogos da teoria cognitivo comportamental, no acompanhamento hospitalar de pacientes e familiares, buscam reestruturar cognições, minimizar estresse com técnicas de relaxamento e fortalecer estratégias de enfrentamento; também estão envolvidos na sistematização de diretrizes que orientam a atuação profissional por meio da construção de protocolos, da 
delimitação de instrumentos, procedimentos e de estratégias baseadas em evidências, buscando a inter-relação da prática e da pesquisa (Azevêdo, 2010; Azevêdo \& Santos, 2011; Gorayeb \& Guerrelhas, 2003; Miyasaki et al., 2006). É possível verificar que os autores definem formas de atuação congruentes com o modelo teórico utilizado, o que permite compreender que as especificidades caracterizam determinada prática ou uma maneira peculiar de contextualizar e definir intervenções psicológicas a serem utilizadas na situação de doença e hospitalização.

Seja qual for a abordagem teórica em Psicologia, existem pontos centrais na atuação do psicólogo no hospital geral. Campos (1995), por exemplo, destaca a necessidade de focalizar a tríade paciente, acompanhante, equipe de saúde. No contato com o paciente, o psicólogo constrói o vínculo terapêutico, mostra-se disponível para a escuta das queixas e demandas, identificando, de forma colaborativa, as situações que provocam sofrimento, visando reorganizar a tensão emocional. Busca-se promover conversações para os acompanhantes, demais familiares e equipe de saúde com o objetivo de mediar o relacionamento e a comunicação destes com o paciente e, por outro lado, atender às demandas emocionais da família.

O atendimento psicológico hospitalar é realizado em locais distintos, como as unidades de internação e ambulatórios. Por causa disso, é preciso considerar as características de cada local, verificando o contexto apropriado para o atendimento, o número de sessões, os horários e o período destinado ao acompanhamento (Azevêdo \& Santos, 2011). O paciente hospitalizado apresenta problemas que são vivenciados em uma situação real de doença e demais agravos da saúde que necessitam de hospitalização, o que exige do psicólogo habilidades para estabelecer vínculo e manter o foco nas demandas centrais, por isso a importância da avaliação e intervenção psicológica.

No processo de avaliação, considera-se relevante o diálogo entre o psicólogo, o paciente, o acompanhante e a equipe de saúde para compartilhar informações. No contexto da Psicologia Pediátrica, por exemplo, a avaliação psicológica englo- ba diversos fatores, como a avaliação de aspectos psicológicos decorrentes da situação de doença e hospitalização, avaliação de estresse e enfrentamento e da dinâmica familiar estabelecida entre criança e acompanhante (Crepaldi et al., 2006). De acordo com as autoras, quando isso ocorre de maneira dinâmica, permite selecionar os instrumentos adequados a serem utilizados no processo de avaliação, assim, escalas psicométricas, entrevistas e a observação representam recursos relevantes. Segundo Pedromônico (2006), a avaliação psicológica hospitalar por meio de protocolo representa um guia que orienta as etapas, auxilia em uma tarefa fundamentada no método científico e possibilita que a equipe de saúde compreenda as atividades desenvolvidas pelo psicólogo.

Os dados obtidos na avaliação servem para descrever informações no prontuário hospitalar e delimitar estratégias de intervenção. Segundo Dias et al. (2003), o planejamento da avaliação e a intervenção efetiva da Psicologia diminuem o tempo de hospitalização do paciente, por considerar que a identificação de distúrbios psicológicos na fase inicial facilita direcionar condutas evitando a evolução dos sintomas. A avaliação psicológica hospitalar tem sido utilizada em pesquisas e é possível verificar, na produção científica, estudos sobre avaliação de estratégias de enfrentamento de crianças com câncer (Moraes \& Enumo, 2008), avaliação da dor em neonatos e crianças (Linhares \& Doca, 2010) e avaliação de estresse em crianças submetidas a cirurgias eletivas após terem participado de um programa de preparação psicológica (Broering \& Crepaldi, 2011). Esses estudos são importantes para o desenvolvimento de novas possibilidades de atuação do psicólogo no hospital geral, por considerar que a avaliação de variáveis específicas permite estruturar as intervenções que serão realizadas, nesse caso, o conjunto de técnicas psicológicas.

A multiplicidade de práticas oriundas das diferentes teorias psicológicas possibilitou estabelecer modelos diferenciados de atuação profissional. É possível destacar os relatos de casos clínicos referentes à intervenção com crianças cardiopatas (Santos \& Guidugli, 2013), ao paciente renal crônico e cardiopata (Almeida, 2013), à gestante à espera de 
um bebê cardiopata (Guidugli, 2013) e a um grupo de pacientes tabagistas (Bueno \& Ismael, 2013). Nessas situações, o psicólogo hospitalar busca investigar a demanda por meio do acolhimento e, ao priorizar a escuta, permite que o paciente verifique as possibilidades de enfrentamento das situações.

De forma específica, a intervenção segue algumas diretrizes dependendo da teoria utilizada. Ao utilizar a abordagem cognitivo comportamental, por exemplo, o psicólogo hospitalar tem o objetivo de auxiliar os pacientes na identificação das situações, dos pensamentos, sentimentos e comportamentos. Busca-se promover, no paciente, a capacidade de reestruturação cognitiva de pensamentos sobre a situação de doença com o propósito de atribuir novos significados e desenvolver a adaptação. Se o paciente relata que não acredita em perspectivas de recuperação, provavelmente surgirão sentimentos de tristeza e comportamentos os quais dificultam sua inserção nos procedimentos do setor, mas se, por outro lado, o psicólogo começa a explorar esse ponto, existe a possibilidade do paciente buscar informações sobre a evolução clínica, manter contato com os profissionais de saúde e verificar que o processo de recuperação implica necessariamente a participação coletiva. As mudanças na maneira de interpretar os eventos possibilitam desenvolver flexibilidade diante das situações vivenciadas.

\section{Considerações Finais}

Os temas tratados neste artigo destacam que a inserção da Psicologia no hospital geral nos EUA e no Brasil ocorreu a partir da década de 1950 e possibilitou o início das práticas psicológicas sobretudo nos hospitais. Foi possível verificar, também, a transformação da área no Brasil. O psicólogo percebeu a importância de estruturar e descrever os procedimentos utilizados no hospital geral, por meio de estudos empíricos. Destacam-se as possibilidades de intervenções fundamentadas em diferentes teorias psicológicas, sendo possível verificar especificidades na atuação do psicólogo no hospital, as quais permitem estruturar um conjunto de práticas

582 direcionadas para a tríade: paciente, família, equipe de saúde. A construção de modelos para a avaliação e intervenção psicológica hospitalar contribuíram de maneira significativa para a delimitação das atividades e para o crescimento contínuo da teoria, prática e pesquisa.

O surgimento da Psicologia Hospitalar contribuiu para edificar a inserção da Psicologia no setor da saúde, porém pode-se dizer que a denominação da área pelo local de intervenção parece pouco adequado. O Brasil avançou muito com o advento do Sistema Único de Saúde (SUS) em 1990, que preconizou a saúde como direito de todos e dever do Estado. Assim, quando se pensa em Psicologia aplicada à saúde, é necessário considerar os diferentes pontos de atenção (primário, secundário e terciário).

A Psicologia brasileira avançou muito ao integrar as equipes de saúde dos diferentes pontos de atenção. Nesse cenário, a Psicologia Hospitalar destina-se a fornecer assistência no que diz respeito ao ponto terciário de atenção, ou seja, privilegia as complexidades média e alta. O que se constata, porém, é que muitos psicólogos brasileiros ainda desconhecem o trabalho realizado no setor da saúde e mesmo aqueles que nele trabalham, muitas vezes, negligenciam o fato de estarem inseridos na rede do SUS. Isso é imprescindível para uma assistência de qualidade. Por fim, é preciso avançar para uma Psicologia da Saúde que contemple todos os pontos de atenção. É importante também que os psicólogos conheçam as políticas públicas em saúde e que considerem participar das equipes para integrar a atenção aos usuários.

\section{Colaboradores}

A.V.S. AZEVÊDO elaborou a concepção, o desenho do estudo, e elaboração do artigo, e M.A. CREPALDI contribuiu no processo de análise e interpretação de dados, e revisão geral do artigo.

\section{Referências}

Almeida, C. P. (2013). Filtrando as emoções: o atendimento psicológico ao paciente renal crônico e cardiopata. In S. M. C. Ismael \& J. X. A. Santos (Orgs.), Psicologia hospitalar: sobre o adoecimento, articulando conceitos com a prática clínica (pp.45-52). São Paulo: Atheneu. 
Almeida, E. C. (2000). O psicólogo no hospital geral. Psicologia: Ciência e Profissão, 20(3), 24-27.

American Psychological Association. (1980). Division 38. Retrieved March 20, 2012, from http://www.apa.org/ about/division/div38.aspx

Angerami-Camon, V. A. (1984). Psicologia hospitalar: a atuação da psicologia no contexto hospitalar. São Paulo: Traço.

Angerami-Camon, V. A. (2002). Psicologia hospitalar, passado, presente e perspectivas. In V. A. Angerami-Camon (Org.), O doente, a psicologia e o hospital (pp.3-27). São Paulo: Cengage Learning.

Angerami-Camon, V. A. (2010). Psicologia hospitalar: teoria e prática (2 ${ }^{a}$ ed.). São Paulo: Cengage Learning.

Amorim, S. F. (2006). Intervenção psicológica no hospital geral. In W. L. Bruscato, C. Benedetti, \& S. R. A. Lopes (Orgs.), A prática da psicologia hospitalar na Santa Casa de São Paulo: novas páginas em uma antiga história (pp.69-80). São Paulo: Casa do Psicólogo.

Azevêdo, A. V. S. (2010). Construção do protocolo de avaliação psicológica hospitalar para a criança queimada. Avaliação Psicológica, 9(1), 99-109.

Azevêdo, A. V. S., \& Santos, A. F. T. (2011). Intervenção psicológica no acompanhamento hospitalar de uma criança queimada. Psicologia: Ciência e Profissão, 31(2), 328-339.

Belar, C. D. (1997). Clinical health psychology: A specialty for the $21^{\circ}$ century. Health Psychology, 16(5), 411-416.

Belar, C. D. (2000). Psychological interventions and health: Critical connections. Psicologia, Saúde \& Doenças, 1(1), 11-17.

Belar, C. D., \& Deardorff, W. W. (1995). Clinical health psychology in medical settings: A practitioner's guidebook $\left(2^{\text {th }}\right.$ ed.). Washington: APA.

Bonaldi, C., Gomes, R. F., Louzada, A. P. F., \& Pinheiro, R. (2007). O trabalho em equipe como dispositivo de integralidade: experiências cotidianas em quatro localidades brasileiras. In R. Pinheiro, R. A. Mattos, \& M. E. B. Barros (Orgs.), Trabalho em equipe sob o eixo da integralidade: valores, saberes e práticas (pp.53-72). Rio de Janeiro: Instituto de Medicina Social.

Bortoletti, F. F., Moron, A. F., Bortoletti Filho, J., Nakamura, U., Santana, R. M., \& Mattar, R. (2007). Psicologia na prática obstétrica: abordagem interdisciplinar. São Paulo: Manole.

Brasil. Ministério da Educação. (2007). Portaria interministerial $n^{\circ} 698$, de 19 de julho de 2007. Nomeação de membros titulares e suplentes da CNRMS. Diário Oficial da União, nº 139, jul. 2007, Seção 2, p. 9. Recuperado em setembro 18, 2014, de http://portal. mec.gov.br/sesu/arquivos/pdf/residencia/portaria_ 698_07.pdf

Brasil. Ministério da Saúde. (2005). Lei n 11.129, de 30 de junho de 2005. Institui a Comissão Nacional de Residência Multiprofissional em Saúde no âmbito do
Ministério da Educação. Recuperado em outubro 20, 2015, de http://www.normaslegais.com.br/legislacao/ resolucao-cnrms-2-2012.htm

Brasil. Ministério da Saúde. (2013). Portaria n 3.390, de 30 de dezembro de 2013. Institui a Política Nacional de Atenção Hospitalar (PNHOSP) no âmbito do Sistema Único de Saúde (SUS), estabelecendo-se as diretrizes para a organização do componente hospitalar da Rede de Atenção à Saúde (RAS). Diário Oficial da União, $n^{\circ}$ 251, dez. 2013, Seção 1, p.170.

Broering, C. V., \& Crepaldi, M. A. (2011). Preparação psicológica e o estresse de crianças submetidas a cirurgias. Psicologia em Estudo, 16(1), 15-23.

Broering, C. V., \& Crepaldi, M. A. (2013). Psychological preparation for surgery: Verbal report of the drawingstory. Estudos de Psicologia (Campinas), 30(3), 367-374. http://dx.doi.org/10.1590/s0103-166X201300030 0006

Bruscato, W. L. (2004). A Psicologia no Hospital da Misericórdia: um modelo de atuação. In W. L. Bruscato, C. Benedetti, \& S. R. A. Lopes (Orgs.), A prática da Psicologia hospitalar na Santa Casa de São Paulo: novas páginas em uma antiga história (pp.17-31). São Paulo: Casa do Psicólogo.

Bueno, P. R. T., \& Ismael, S. M. C. (2013). O grupo como facilitador no atendimento ao paciente tabagista. In S. M. C. Ismael \& J. X. A. Santos (Orgs.), Psicologia hospitalar: sobre o adoecimento, articulando conceitos com a prática clínica (pp.53-63). São Paulo: Atheneu.

Campos, T. C. P. (1995). Psicologia hospitalar: a atuação do psicólogo em hospitais. São Paulo: Epu.

Chiattone, H. B. C. (2006). A significação da psicologia no contexto hospitalar. In V. A. A. Camon (Org.), Psicologia da saúde: um novo significado para a prática clínica (pp.73-167). São Paulo: Cengage Learning.

Conselho Federal de Psicologia. (2000). Resolução n014/00, de 20 de dezembro de 2000. Institui o título profissional de especialista em Psicologia e dispõe sobre normas e procedimentos para seu registro. Brasília: Autor.

Conselho Federal de Psicologia. (2001). Resolução nº 02/2001, de 10 de março de 2001. Altera e regulamenta a resolução CFP nº 014/00 que institui o título profissional de especialista em Psicologia e o respectivo registro nos Conselhos Regionais. Brasília: Autor.

Conselho Federal de Psicologia. (2007). Resolução n 015/ 2007, de 19 de setembro de 2007. Dispõe sobre o credenciamento de cursos de Residência em Psicologia na área de Saúde e revoga a Resolução CFP n 009/ 2000. Brasília: Autor.

Conselho Regional de Psicologia. (2007). Manual de psicologia hospitalar: Coletânea ConexãoPsi - Série Técnica. Curitiba: Autor.

Crepaldi, M. A. (1999a). Programas de hospitalização conjunta: integrando os pais em enfermarias pediátricas. Temas em Psicologia, 7(2), 157-173. 
Crepaldi, M. A. (1999b). Hospitalização na infância: representações sociais da família sobre a doença e a hospitalização de seus filhos. Taubaté: Cabral.

Crepaldi, M. A., Gonçalves, J. R., \& Moré, C. L. O. O. (2011). A história do departamento de psicologia da UFSC na área da saúde. In Conselho Regional de Psicologia - $12^{\text {a }}$ região (Org.), Memórias da Psicologia Catarinense (pp.15-20). Florianópolis: CRP.

Crepaldi, M. A., Rabuske, M. M., \& Gabarra, L. M. (2006). Modalidades de atuação do psicólogo em psicologia pediátrica. In M. A. Crepaldi, M. B. M. Linhares, \& G. B. Perosa (Orgs.), Temas em Psicologia pediátrica (pp.1356). São Paulo: Casa do Psicólogo.

Dias, R. R., Baptista, M. N., \& Baptista, A. S. D. (2003). Enfermaria de pediatria: avaliação e intervenção psicológica. In M. N. Baptista \& R. R. Dias (Orgs.), Psicologia hospitalar: teoria, aplicações e casos clínicos (pp.59-69). Rio de Janeiro: Guanabara Koogan.

Dorken, H. (1993). The hospital private practice of Psychology: CHAMPUS 1981-1991. Professional Psychology Research and Practice, 24(4), 409-417.

Enright, M. F., Resnick, R. J., DeLeon, P. H., Sciara, A. D., \& Tanney, F. (1990). The practice of psychology in hospital settings: Psychology in the public forum. Americam Psychologist, 45(9), 1059-1065.

Enright, M. F., Resnick, R. J., Ludwigsen, K. R., \& DeLeon, P. H. (1993). Hospital practice: Psychology's call to action. Professional Psychology Research and Practice, 24(2), 135-141.

Gimenes, M. G., Magui, M. M. M. J. C., \& Carvalho, V. A. (2006). Um pouco da história da psico-oncologia no Brasil. In V. A. Angerami-Camon (Org.), Psicologia da saúde: um novo significado para a prática clínica (pp.47-71). São Paulo: Cengage Learning.

Gorayeb, R. (2010). Psicologia da saúde no Brasil. Psicologia: Teoria e Pesquisa, 29(Vol. Esp.), 115-122.

Gorayeb, R., \& Guerrelhas, F. (2003). Sistematização da prática psicológica em ambientes médicos. Revista Brasileira de Terapia Comportamental e Cognitiva, 5(1), 11-19.

Guidugli, S. K. K. (2013). Coração aflito: a gestante à espera do bebê cardiopata. In S. M. C. Ismael \& J. X. A. Santos (Orgs.), Psicologia hospitalar: sobre o adoecimento, articulando conceitos com a prática clínica (pp.27-34). São Paulo: Atheneu.

Ismael, S. M. C. (2005). Temas de prevenção, ensino e pesquisa que permeiam o contexto hospitalar. São Paulo: Casa do Psicólogo.

Kerbauy, R. R. (1999). Comportamento e saúde: explorando alternativas. Santo André: ARBytes.

Knobel, E., Andreoli, P. B. A., \& Erlichman, M. R. (2008). Psicologia e humanização: assistência aos pacientes graves. São Paulo: Atheneu.

Leahy, R. L., Tirch, D., \& Napolitano, L. A. (2013). Regulação emocional em psicoterapia: um guia para o terapeuta cognitivo-comportamental. Porto Alegre: Artmed.

Linhares, M. B. M., \& Doca, F. N. P. (2010). Dor em neonatos e crianças: avaliação e intervenções não farmacológicas. Temas em Psicologia, 18(2), 307-325.

Matarazzo, J. D. (1980). Behavioral health and behavioral medicine: Frontiers for a new health Psychology. American Psychologist, 35(9), 807-817.

Mattos, R. A. (2003). Integralidade e a formulação de políticas específicas de saúde. In R. Pinheiro \& R. A. Mattos (Orgs.), Construção da integralidade: cotidiano, saberes e práticas em saúde (pp.45-99). Rio de Janeiro: Universidade Estadual do Rio de Janeiro.

Mettel, E. T. P. L. (2007). Proposta de concessão do título de professor emérito, pela Universidade de Brasília, à Profa. Dra. Thereza Pontual de Lemos Mettel. Psicologia: Teoria e Pesquisa, 23(Num. Esp.), 125-129.

Miyasaki, M. C. O. S., Domingos, N. A. M., \& Valério, N. I. (2006). Psicologia da saúde: pesquisa e prática. São José do Rio Preto: THS/Arantes.

Moraes, E. O., \& Enumo, S. R. F. (2008). Estratégias de enfrentamento da hospitalização em crianças avaliadas por instrumento informatizado. Psico-USF, 13(2), 221-231.

Moré, C. L. O. O., Crepaldi, M. A., Gonçalves, J. R., \& Menezes, M. (2009). Contribuições do pensamento sistêmico à prática do psicólogo no contexto hospitalar. Psicologia em Estudo, 14(3), 465-473.

Nogueira-Martins, L. A., \& Frenk, B. (1980). A atuação do profissional de saúde mental no hospital de ensino: a interconsulta médico-psicológica. Boletim de Psicologia, 3(1), 30-37.

Pate, W. E., \& Kohut, J. L. (2003). Results from a national survey of psychologists in medical school settings. Journal of Clinical Psychology in Medical Settings, 12(3), 85-91.

Pedromônico, M. R. M. (2006). A relevância da avaliação psicológica na clínica pediátrica. In M. A. Crepaldi, M. B. M. Linhares, \& G. B. Perosa (Orgs.), Temas em Psicologia pediátrica (pp.81-107). São Paulo: Casa do Psicólogo.

Queiroz, A. M. (1958). Some psychological contributions to the clinical treatment of the asthmatic child. Acta Allergologica, 12(6), 396-406.

Queiroz, A. M. (1961). Os aspectos psicológicos da criança enferma. Revista de Psicologia Normal e Patológica, 7(1-3), 463-468.

Romano, B. W. (1990). A prática da psicologia nos hospitais. São Paulo: Pioneira Thomson Learning.

Romano, B. W. (1999). Princípios para a prática da psicologia clínica em hospitais. São Paulo: Casa do Psicólogo.

Romano, B. W. (2001). Psicologia e cardiologia: encontros possíveis. São Paulo: Casa do Psicólogo. 
Romano, B. W. (2008). Manual de psicologia clínica para hospitais. São Paulo: Casa do Psicólogo.

Santos, F. M. S., \& Jacó-Vilela, A. M. (2009). O psicólogo no hospital geral: estilos e coletivos de pensamento. Paidéia, 19(43), 189-197.

Santos, J. X. A., \& Guidugli, S. K. N. (2013). Acompanhamento psicológico aos pequenos pacientes cardiopatas. In S. M. C. Ismael \& J. X. A. Santos (Orgs.), Psicologia hospitalar: sobre o adoecimento, articulando conceitos com a prática clínica (pp.35-44). São Paulo: Atheneu.

Sebastiani, R. W. (2006). Histórico e evolução da psicologia da saúde numa perspectiva Latino-Americana. In V. A. A. Camon (Org.), Psicologia da saúde: um novo significado para a prática clínica (pp.221-222). São Paulo: Thomson Learning.

Silva, L. P. P. (2006). O percurso histórico do serviço de psicologia do Hospital de Clínicas de Porto Alegre
(Dissertação de mestrado não-publicada). Universidade Federal do Rio Grande do Sul, Porto Alegre.

Simonetti, A. (2004). Manual de psicologia hospitalar: o mapa da doença. São Paulo: Casa do Psicólogo.

Straub, R. O. (2008). Psicologia da saúde. Porto Alegre: Artmed.

Valle, E. R. M. (2001). Possibilidades de atuação em psico-oncologia pediátrica. In E. R. M. Valle (Org.), Psico-oncologia pediátrica (pp.77-85). São Paulo: Casa do Psicólogo.

Zannon, C. M. C. L. (1981). O comportamento de crianças hospitalizadas e a rotina hospitalar: subsidios para atuação do psicólogo junto à equipe de pediatria (Tese de doutorado não-publicada). Universidade de São Paulo.

Recebido: julho 17, 2014

Versão final: fevereiro 3, 2015

Aprovado: junho 11, 2015 
